\title{
Spontaneous bladder rupture: the images before and after
}

\author{
Mitsumasa Okano ${ }^{1}$, Toshihiko Oshita ${ }^{1}$, Kazunori Otsui ${ }^{1}$, and Kazuhiko Sakaguchi ${ }^{1}$ \\ ${ }^{1}$ Kobe University Graduate School of Medicine
}

November 13, 2020

\begin{abstract}
Nontraumatic bladder rupture is a rare cause of abdominal pain. We presented a case of spontaneous bladder rupture following acute cystits.
\end{abstract}

\section{Clinical Image}

Spontaneous bladder rupture: the images before and after

Mitsumasa Okano, MD, PhD, Toshihiko Oshita, MD, PhD,

Kazunori Otsui, MD, PhD, Kazuhiko Sakaguchi, MD, PhD

Division of General Internal Medicine, Department of Internal Medicine,

Kobe University Graduate School of Medicine

Corresponding author: Mitsumasa Okano, Division of General Internal Medicine,

Department of Internal Medicine, Kobe University Graduate School of Medicine

7-5-1, Kusunoki-cho, Chuo-ku, Kobe 650-0017, Japan.

Tel:+81-78-382-6596, Fax:+81-78-382-6597

E-mail: mokano@med.kobe-u.ac.jp

Key words: spontaneous bladder rupture, acute cystitis, bladder diverticulum,

CT scan, retrograde cystography

\section{Key Clinical Message}

For patients with worsened abdominal pain following acute cystitis, we should consider the complication of bladder rupture. A CT scan is a useful first-line modality when evaluating for a suspected bladder rupture.

A 50-year-old woman, taking prednisolone for 20 years to her autoimmune hepatitis, presented with suprapubic pain during urination. She was diagnosed as acute cystitis based on pyuria and bacteriuria. Her initial CT scan showed bladder diverticulum (Figure 1A ). Next day, she was admitted to our hospital because of worsened symptom despite of antibiotic therapy. Physical examination revealed suprapubic tenderness with guarding. Laboratory findings showed elevated inflammation markers and serum creatinine level. Repeated CT scan showed the fluid collection in anteroventral portion of the bladder with thickening of pelvic peritoneum (Figure 1B ). Her retrograde cystography revealed contrast material moving outside the bladder into extraperitoneal space (Figure 1C ). Perforation site of the diverticulum was not evident by 
cystoscopy. She was diagnosed as spontaneous extraperitoneal bladder rupture, which led to pelvic peritonitis and pseudo-renal failure due to creatinine reabsorption across the peritoneal membrane. The insertion of urethral catheter improved her symptom dramatically.

Pathological bladder wall fragility and increased intra-bladder pressure are recognized as risk factors for nontraumatic bladder rupture. In our case, sequential CT images suggested excessive bladder retention caused by acute cystitis provoked bladder rupture on the background of bladder-wall fragility due to longterm steroid use[1] and/or diverticulum.[2]

\section{Acknowledgements}

None.

\section{Conflict of Interest}

None declared.

\section{Author Contributions}

MO: drafted the manuscript. TO, KO and KS: supervised it. All authors read and approved the final manuscript.

\section{References}

1. Ribeiro, G. S., D. B. Souza, C. M. Cortez, D. Silva, W. S. Costa and F. J. Sampaio. 2014. Effects of prepubertal corticosterone treatment on urinary bladder. Acta Cir Bras. 29 Suppl 3:55-59.

2. Kodama, K., Y. Takase and K. Saito. 2016. Extraperitoneal Rupture of a Bladder Diverticulum and the Role of Multidetector Computed Tomography Cystography. Urol Case Rep. 9:30-32.

\section{Figure Legend}

\section{Figure 1}

A, Initial CT scan showed bladder diverticulum (arrow) and thickened bladder wall with stranding in the surrounding fat.

B, Repeated CT scan showed fluid collection in anteroventral portion of the bladder (asterisk) with thickening of pelvic peritoneum (arrows).

C, Retrograde cystography showed contrast material moving outside the bladder (arrows). 

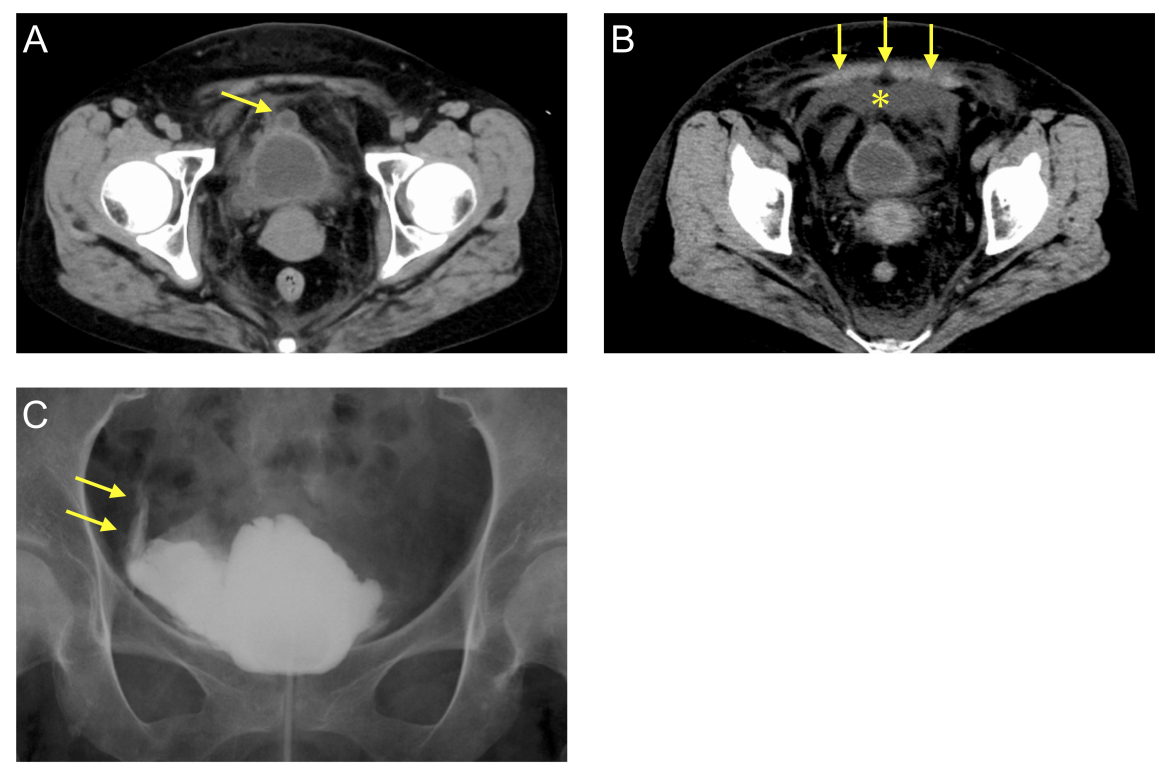

Figure 1 